\title{
Modal Analysis of Equal Spacing around Sun Gear Double Power Branch System
}

\author{
Xigui Wang ${ }^{1}$, Yongmei Wang ${ }^{2}$, Xuezeng Zhao ${ }^{3}$ \\ ${ }^{1}$ Harbin Institute of Technology, Mechatronics School, No.92, Xidazhi Street, Nangang District, Harbin 150001, China \\ 703 Research of Institute, Department of Marine Gear Transmission System, No.35, Honghu Road, Daoli District, Harbin 150078, China \\ ${ }^{2}$ Heilongjiang Institute of Technology, School of Motorcar Engineering, No.999, Hongqi Street, Daowai District, Harbin 150036, China \\ ${ }^{3}$ Harbin Institute of Technology, Mechatronics School, No.92, Xidazhi Street, Nangang District, Harbin 150001, China
}

\begin{abstract}
Modal analysis techniques are applied to characterize the planar dynamic characteristics of two planetary gears. Rotational and translational vibrations of the sun gear, star gear, and planet gears are displayed in planetary transmission system. This paper obtained star and planetary gear system vibration mode, coupling vibration modal under different classes of frequencies, and are observed in the analysis and compared to the results from planetary transmission system land based integration test. The first level of star gear and second planetary gears in the respective gear transmission around sun gear equal spacing layout, regularity modal system vibration is obvious.
\end{abstract}

Keywords: Planet gears, Sun gear, Equal spacing, Dynamic characteristics

\section{Introduction}

While planetary gearboxes usually have unequally spaced planets, some planetary gears have equally spaced planets due to the limitations of assembly conditions or special applications [1-3].

Considering subject requirements, Table 1-2 summarizes the basic parameters of two stage power branch at all levels of double planetary composite transmission system for underwater device. First hypothesis star and second planetary gear in the gear train around the sun gear spacing, planet and star gear structure are same, two star gear and planetary gear bearing support stiffness are equal, engaging star or planet and sun gear meshing stiffness are equal, star or planet gear and ring gear stiffness are equal. As shown in Table 2, multiplicity table numbers in parentheses is the inherent frequency, natural frequency is not marked as a single.

In the literature, many studies exist on the mode shapes, Parker and $\mathrm{Wu}$ [4] analytically investigated the vibration modes of planetary gears with unequally spaced planets and an elastic ring gear. Guo and Parker [5] studied the modal properties of compound planetary gears using a model with only rotational degrees of freedom. Recently, Cooley and Parker examined gyroscopic system eigenvalue behavior in high-speed planetary gears [6], which can take advantage of the modal properties derived in this work.

\section{System Vibration Mode}

The modal shapes are fundamental when dealing with an existing vibration problem or designing new systems to avoid resonant vibration, as gear engineers routinely need to do. This paper provides detailed and rigorously derived properties of the modes for planetary gears with equally spaced planets. The work provides knowledge engineers can use in practice as well as modal properties critical to further research on resonant vibration response, non-linearity, diagnostics, and the like.

Table 1: System parameters

\begin{tabular}{|c|l|}
\hline & $k_{s p}^{I}=22.478 \times 10^{9} ;$ \\
& $k_{r p}^{I}=3.439 \times 10^{9} ;$ \\
Meshing stiffness (N/m) & $k_{s p}^{I I}=20.497 \times 10^{9} ;$ \\
& $k_{r p}^{I I}=2.947 \times 10^{9}$ \\
\hline & $k_{r}^{I}=k_{c}^{I I}=k_{r}^{I I}=k_{s}^{I I}=$ \\
& $k_{p}^{I}=k_{p}^{I I}=1.0 \times 10^{10}$ \\
Support stiffness (N/m) & $k_{s}^{I}=100$ \\
& $k_{r s}^{I 2}=0$ \\
\hline Shaft torsional Stiffness (N.m/rad) & $k_{r s u}^{12}=2.0 \times 10^{8}$ \\
\hline & $\alpha_{s m}=\alpha_{r m}=$ \\
Pressure angle ( $\left.{ }^{0}\right)$ & $\alpha_{s n}=\alpha_{r n}=20$ \\
\hline Helix angle ( $\left.{ }^{0}\right)$ & $\beta_{b m}=\beta_{b n}=25.23$ \\
\hline
\end{tabular}

In Tab. 2, Quality (Q) (kg), Equivalent moment inertia (Emi) $(\mathrm{kg})$, Base circle diameter $(\mathrm{Bcd})(\mathrm{mm})$, Number teeth $(\mathrm{Nt})$.

Table 2: System parameters

\begin{tabular}{|c|c|c|c|c|c|c|}
\hline \multirow{2}{*}{} & \multicolumn{2}{|c|}{ Sun gear } & \multicolumn{2}{c|}{ Ring gear } & \multicolumn{2}{c|}{ Star/planet } \\
\cline { 2 - 7 } & $I$ & $I I$ & $I$ & $I I$ & $I$ & $I I$ \\
\hline$Q$ & 98 & 395 & 256 & 645 & 298 & 645 \\
\hline$E m i$ & 52 & 198 & 243 & 612 & 148 & 322 \\
\hline$B c d$ & 384.6 & 476. & 1698 & 1697 & 656 & 608 \\
\hline$N t$ & 43 & 53 & 183 & 183 & 72 & 67 \\
\hline
\end{tabular}

\subsection{Star gear system vibration mode}

The mode shapes calculated and summed up three kinds of the system vibration modals: star gear vibration modal, planetary gear vibration modal and modal coupling [7-9]. 


\section{International Journal of Science and Research (IJSR) \\ ISSN (Online): 2319-7064}

Index Copernicus Value (2013): 6.14 | Impact Factor (2014): 5.611

The first stage star gear in planetary gear vibration system, when no vibration, called this mode is the star gear vibration mode [10-12]. These variation laws:

(1) Star gear numbers are certain, the number of planetary gear is changed, the natural frequency of the system without change. The modal properties of planetary gears with equally spaced based on the discrete and lumped-parameter models.

(2) The number of planetary gear is constant, with the increase of star gear numbers, the system variation of natural frequency is not significant [13-15]. For equally spaced planets with even $\mathrm{N}$, all the modes fall into two types: $\mathrm{M}>3$ rotational modes and M-3 translational modes. The total number of rotational and translational modes equals the total degrees of freedom, so no other mode types are possible. In these frequencies, vibration central is component of star gear, star formation rate, with natural frequency Table 3 corresponding to $934.8 \mathrm{~Hz}$, $1259.6 \mathrm{~Hz}$ and $2474.7 \mathrm{~Hz}$; and a single frequency, center star gear components is only torsional vibration, gear deformation is the same as in Table 3, and the corresponding frequency is $6638.8 \mathrm{~Hz}$.

Table 3: Natural frequencies of the system with star gear numbers $\mathrm{M}=4$

\begin{tabular}{|c|c|c|c|}
\hline & $N=3$ & $N=4$ & $N=5$ \\
\hline $\begin{array}{l}\text { Star gear system } \\
\text { vibration modal } \\
\text { frequency }(\mathrm{Hz})\end{array}$ & $\begin{array}{c}934.8 \\
1259.6 \\
1533.4 \\
1545.2 \\
2474.7 \\
3729.6 \\
6638.8\end{array}$ & $\begin{array}{c}934.8 \\
1259.6 \\
1533.4 \\
1545.2 \\
2474.7 \\
6638.8\end{array}$ & $\begin{array}{c}934.8 \\
1259.6 \\
1533.4 \\
1545.2 \\
2474.7 \\
3729.6 \\
6638.8\end{array}$ \\
\hline $\begin{array}{l}\text { Planetary gear } \\
\text { vibration modal } \\
\text { frequency }(\mathrm{Hz})\end{array}$ & $\begin{array}{c}516.8(2) \\
707.9(2) \\
1043.1(2) \\
1152.3(2) \\
1544.2(2) \\
2696.7(2)\end{array}$ & $\begin{array}{c}494.3(2) \\
726.9(2) \\
972.7 \\
1027.9(2) \\
1163.8(2) \\
1173.1 \\
1664.7(2) \\
2015.6 \\
3205.8(2)\end{array}$ & $\begin{array}{l}473.5(2) \\
743.2(2) \\
972.7(2) \\
1016.8(2) \\
1179.1(2) \\
1173.1(2) \\
1778.8(2) \\
2015.6(2) \\
3427.8(2)\end{array}$ \\
\hline $\begin{array}{c}\text { Coupling } \\
\text { vibration modal } \\
\text { frequency }(\mathrm{Hz})\end{array}$ & $\begin{array}{l}486.1 \\
691.1 \\
797.7 \\
826.8 \\
864.8 \\
927.6 \\
941.2 \\
972.7 \\
1063.2 \\
1078.8 \\
1116.9 \\
1132.7 \\
1301.4 \\
1376.4 \\
1761.6 \\
3389.7\end{array}$ & $\begin{array}{l}470.8 \\
690.9 \\
825.6 \\
826.7 \\
864.7 \\
927.3 \\
939.8 \\
975.1 \\
1063.1 \\
1078.7 \\
1133.2 \\
1141.3 \\
1300.8 \\
1440.6 \\
1763.1 \\
3717.9 \\
3729.6 \\
3729.6\end{array}$ & $\begin{array}{l}455.7 \\
690.9 \\
826.8 \\
843.6 \\
864.6 \\
927.2 \\
939.7 \\
969.8 \\
1063.1 \\
1078.7 \\
1133.2 \\
1174.1 \\
1300.8 \\
1503.3 \\
1763.1 \\
4017.8\end{array}$ \\
\hline
\end{tabular}

\subsection{Planetary gear system vibration mode}

Second stage planetary gear train vibration, when first stage star gear train without vibration, called the modal vibration mode is planetary gear system [16-18]. The change rules:

(1) Star gear numbers are certain, with the increase of planetary gear numbers, the natural frequency of system overall trend is increasing; the natural frequency of two order modes is decreasing. When $N / 2$ is even, $N / 2-1$ is odd. Accordingly, half in $N>3$ are odd and the other half are even. Thus, planet modes contain odd nodal diameter components, and they evolve into translational modes; planet modes evolve into rotational modes, where the additional modes come from the distinct planet modes. At these frequencies, star gear, planetary gears and coupling vibration modal frequency corresponding respectively are $972.7 \mathrm{~Hz}$, $1173.1 \mathrm{~Hz}$ and $2015.6 \mathrm{~Hz}$, as shown in Table 3.

(2) When the planetary gears numbers are fixed, the number change of star gear and the system natural frequency remain unchanged, the total numbers are $3 \times(N+1)$.

\subsection{System Coupling Vibration Modal}

The first stage star gear train coupling, when second stage planetary gear system have vibration, which called the modal coupling vibration mode [19]. These change rules are:

(1) When star gear numbers are certain, planetary gear numbers are changed, the system inherent frequency change is small, the system without repeated root [20]

(2) When planetary gear numbers are certain, star gear numbers are changed, the system natural frequency is showed an overall increasing trend [21].

(3) Following the change of star gear or planetary gears numbers, natural frequency and vibration modal and star shaped gear coupling vibration mode is shifting [22].

A typical vibration $(M=N=4)$ mode component, as shown in Figure 1, 2 and 3. Solid line represents the actual location component after exercise, dotted lines indicates the location of the original assembly. In order to make these pictures clear and concise, gear ring and a planet frame are given only translational, torsional deformations do not mention.

Details of the model in Fig. 1 including typical vibration modal of star gear train, in Fig. 1 a), b) ,c), natural frequencies of vibration mode corresponding respectively are $934.8 \mathrm{~Hz}$, $1259.6 \mathrm{~Hz}$ and $2474.7 \mathrm{~Hz}$, when their multiplicities are $\mathrm{M}-3$, Fig. $1 \mathrm{~d}$ ) corresponding to single frequency is $6638.8 \mathrm{~Hz}$. 


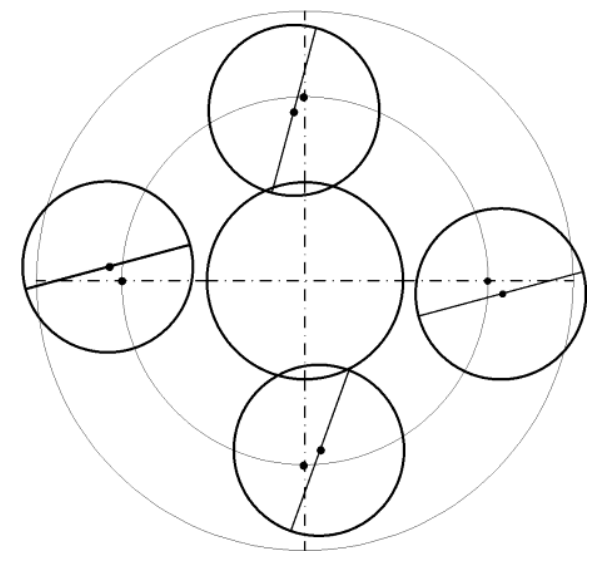

a) Star system mode $(934.8 \mathrm{~Hz})$

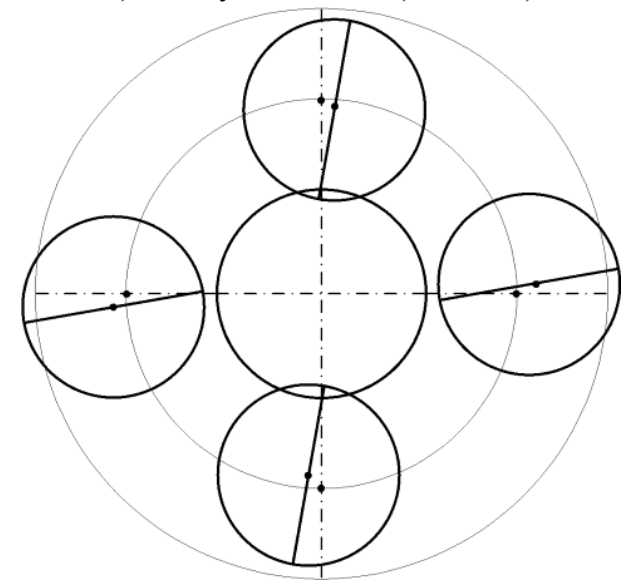

b) Star system mode $(1259.6 \mathrm{~Hz})$

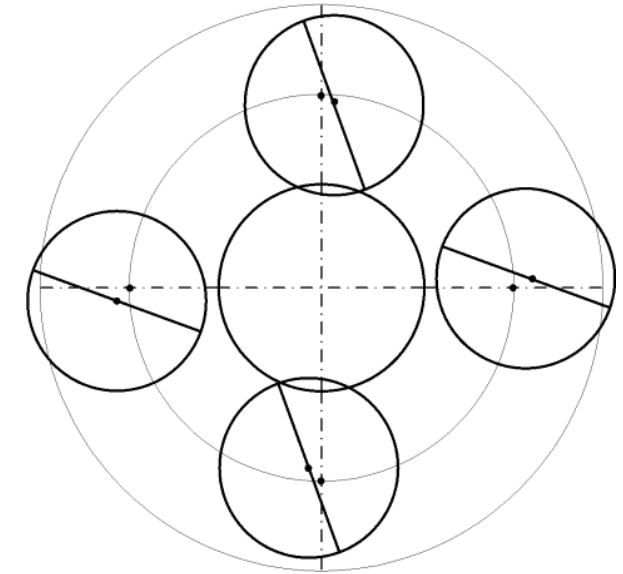

c) Star system mode $(2474.7 \mathrm{~Hz})$

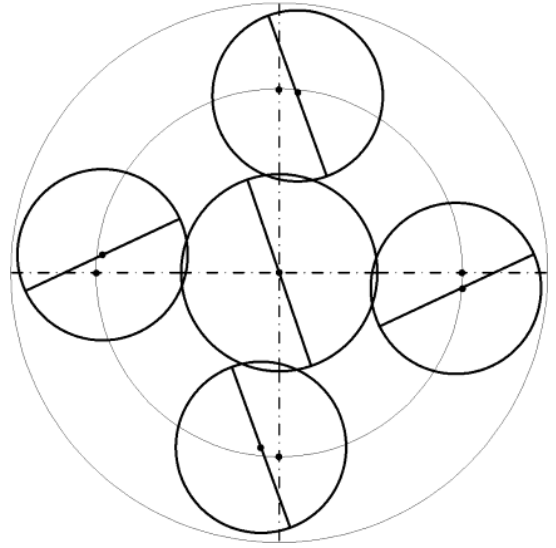

d) Star system mode $(6638.8 \mathrm{~Hz})$

Figure 1: Typical vibration mode of star system $(M=N=4)$

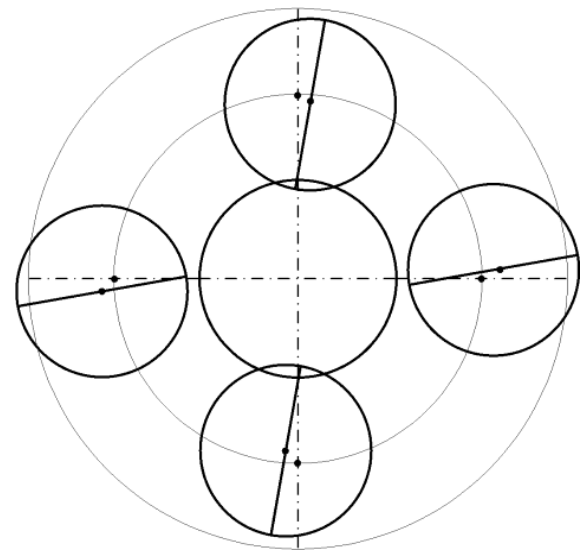

a) Planet system mode $(972.7 \mathrm{~Hz})$

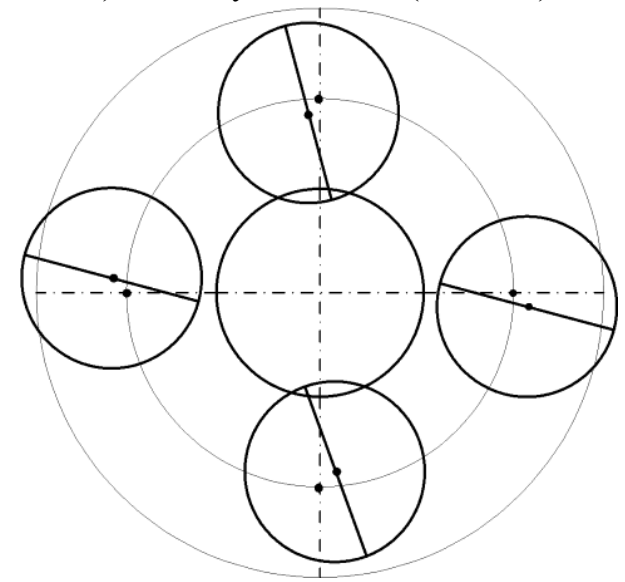

b) Planet system mode $(1173.1 \mathrm{~Hz})$

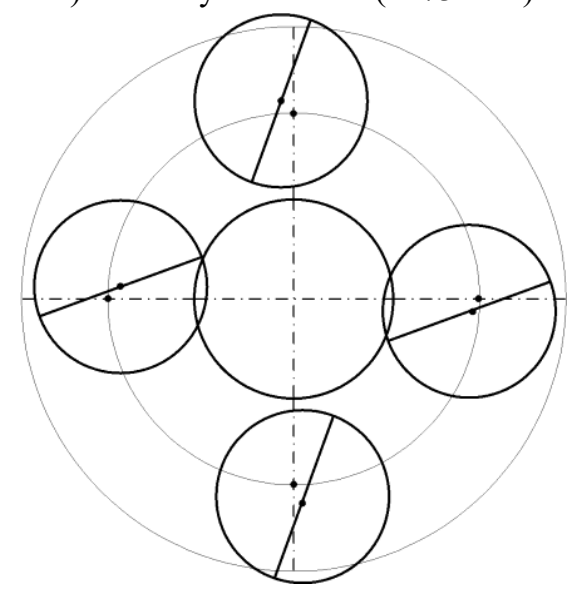

c) Planet system mode $(2015.6 \mathrm{~Hz})$

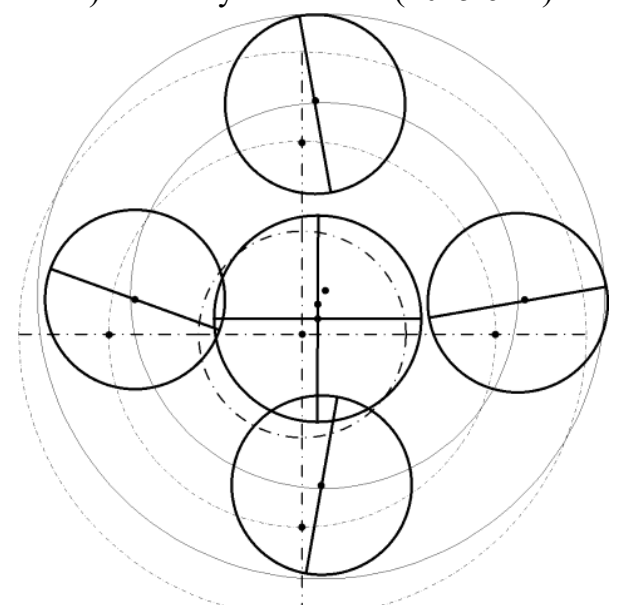

d) Planet system mode $(494.3 \mathrm{~Hz})$

\section{Volume 4 Issue 11, November 2015}




\section{International Journal of Science and Research (IJSR) \\ ISSN (Online): 2319-7064}

Index Copernicus Value (2013): 6.14 | Impact Factor (2014): 5.611

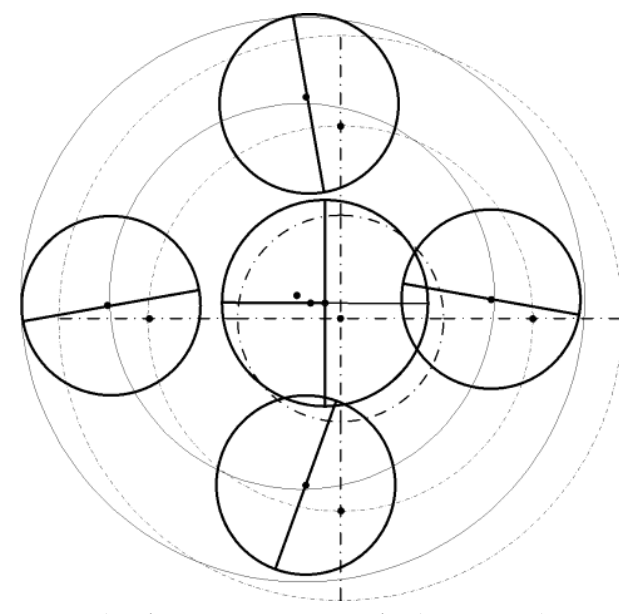

e) Planet system mode $(494.3 \mathrm{~Hz})$

Figure 2: Typical vibration mode of planet system $(M=N=4)$

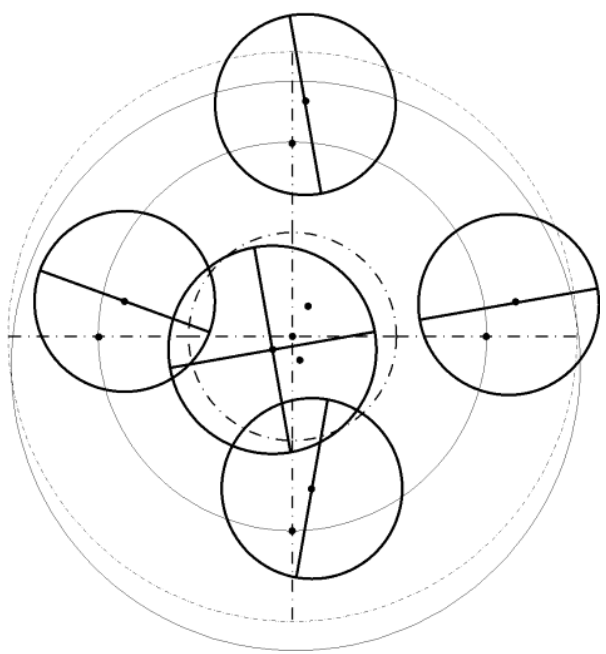

a) Star system $(470.8 \mathrm{~Hz})$

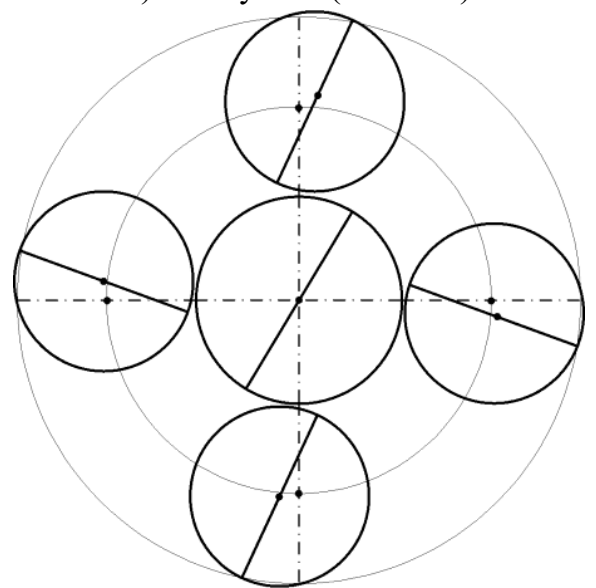

b) Planet system $(470.8 \mathrm{~Hz})$

Figure 3: Typical coupling vibration mode $(M=N=4)$

Fig. 1 a), b) ,c) can be seen, when star gear no moving component, deformation in three directions with a certain proportion, as Fig. 1 d) shown mode corresponding is single frequency, the central component of star gear train only torsional movement, deformation is the same.

As Fig. 2 a), b), c) shown natural frequencies of vibration mode corresponding respectively are $972.7 \mathrm{~Hz}, 1173.1 \mathrm{~Hz}$ and $2015.6 \mathrm{~Hz}$, when their multiplicities are $N-3$, from Fig. $2 \mathrm{~d}$ ) and e) seen vibration mode of a double root inherent frequency corresponds to $494.3 \mathrm{~Hz}$.

Fig. 3 defined the mode of star gear and planetary gear train components coupled vibration mode. Coupled vibration mode, each component has star gear and planetary gear train vibration type, vibration type of star gear train as shown in Fig. 3 a), vibration type of planetary gear system as shown in Fig. 3 b).

As shown in Fig.3 a), center star gear train components not only translational motion, but also torsion with the three movement direction, but vibration characteristics is no regularity. Planetary gear vibration shown in Fig. 3 b), center component is only torsion deformation.

Can be seen from the above analysis, when the interaction between the two stages, the first stage star gear train is maximum. Although the second stage planetary gear train components mode still has certain regularity, but planetary gear vibration modal is diversity.

The next step analysis is the modal characteristics and each vibration mode relation, according to the vibration characteristics of the simplified system value problem, the modal characteristics is corresponding.

\section{Characteristics Analysis}

\subsection{Star Gear Vibration Modal Analysis}

The star gear vibration mode has the following characteristics: (1) In planetary gear system, sun gear, ring gear, planetary gear and a planetary frame vibration displacement respectively are zero, $p_{h}^{I I}=0, h=c, r, s, p_{n}^{I I}=0, n=1 \cdots N$.

(2) When there is a single frequency, the expression of the deformation of center star gear train components gives, $p_{h}^{I}=\left[0,0, u_{h}\right], h=r, s$.

(3) When there are three natural frequency began to appear in time, and the repeated root number is $M-3$.

First inspects the modal characteristics of three natural frequencies with multiple root of the star gear, the expression of the modal shape:

$$
f_{i}=\left[0,0, p_{1}^{I}, \ldots, w_{m} p_{1}^{I}, 0,0,0,0, \ldots, 0\right]^{T}
$$

When modal planetary gear vibration modal type and single stage planetary gear are similar, the equations become respectively.

$$
\begin{aligned}
& k_{r p}^{I} \cos ^{2} \beta_{b m}\left(\zeta_{1}^{I} \sin \alpha_{r m}-\eta_{1}^{I} \cos \alpha_{r m}-u_{1}^{I}\right) \\
& \sum w_{m}\left[\begin{array}{c}
-\sin \psi_{r m} \\
\cos \psi_{r m} \\
1
\end{array}\right]=0
\end{aligned}
$$




$$
\begin{aligned}
& k_{s p}^{I} \cos ^{2} \beta_{b m}\left(\zeta_{1}^{I} \sin \alpha_{s m}+\eta_{1}^{I} \cos \alpha_{s m}-u_{1}^{I}\right) \\
& \sum w_{m}\left[\begin{array}{c}
\sin \psi_{s m} \\
-\cos \psi_{s m} \\
-1
\end{array}\right]=0 \\
& \left(K_{p m}^{m}-\omega_{i}^{2} M_{p}^{I}\right) w_{m} p_{l}^{I}=0, m=1,2, L, M
\end{aligned}
$$

The remaining equations in (2) vanish. Similarly, (3) and (4) reduce to the following equations.

$$
\begin{aligned}
& \sum w_{m} \cos \psi_{m}=0 \\
& \sum w_{m} \sin \psi_{m}=0 \\
& \sum w_{m}=0
\end{aligned}
$$

Where $\phi_{i}$ is multiplicity of eigenvalues for the equations $M-3, p_{1}^{I}$ equations from Eq. (4) for the planet motions.

Eq. (5) can be regarded as the freedom system of homogeneous linear equations linear transform results, have M-3 group nonzero linear independent solutions.

With the modal expressions (4) and (5) and straight forward manipulation, the following modal characteristics equations.

$$
\lambda_{l}=\left(k_{p m}^{I}+2 k_{m}^{I} \cos ^{2} \alpha_{m} \cos ^{2} \beta_{b m}\right) / m_{p}^{I}
$$

Substituting (4) and (5) into (6), determining by $\lambda_{2}, \lambda_{3}$ equations for star mode system respectively, Eq. 7 is discussed below.

$$
\begin{aligned}
& \lambda^{2}-\left[\frac{\left(k_{p u m}^{I}+2 k_{m}^{I} \cos ^{2} \beta_{b m}\right)\left(r_{p}^{I}\right)^{2}}{I_{p}^{I}}+\right. \\
& \left.\frac{k_{p m}^{I}+2 k_{m}^{I} \cos ^{2} \alpha_{m} \cos ^{2} \beta_{b m}}{m_{p}^{I}}\right] \lambda+ \\
& \frac{\left[k_{p m}^{I} k_{p u m}^{I}+2 k_{m}^{I} \cos ^{2} \beta_{b m} k_{p m}^{I}\left(r_{p}^{I}\right)^{2}\right]}{m_{p}^{I} I_{p}^{I}}+ \\
& \frac{\left[2 k_{m}^{I} \cos ^{2} \beta_{b m} k_{p u m}^{I} \sin ^{2} \alpha_{m}\left(r_{p}^{I}\right)^{2}\right]}{m_{p}^{I} I_{p}^{I}}=0
\end{aligned}
$$

Where $\lambda_{1}, \lambda_{2}$ and $\lambda_{3}$ equations, these vibration mode are corresponding.

When $M>3$, a star gear mode calculated, pure radial, tangential and rotation of the star pure torsional motion, and $\alpha_{m}=0$.

Eq. (2) represents the applied force and the star gear toothed ring on the resultant moment, as can be seen, applied force and the ring gear of the resultant moment is zero. By the same Eq. (3) shown, applied force and the sun gear force moment is zero.

When $M$ is even, where $w_{m}=(-1)^{m}$.

Substitution of (3) and (4) into (5), (6) and (7) generates the following three equations.

$$
\begin{aligned}
& {\left[k_{r u}^{I}+\frac{k_{r s u}^{I 2}}{\left(r_{r}^{I}\right)^{2}}+M k_{r p}^{I}-\omega_{i}^{2} \frac{I_{r}^{I}}{\left(r_{r}^{I}\right)^{2}}\right] u_{r}^{I}-} \\
& M k_{r p}^{I}\left(u_{1}^{I}-\zeta_{1}^{I} \sin \alpha_{r m}+\eta_{1}^{I} \cos \alpha_{r m}\right)=0
\end{aligned}
$$

$$
\begin{aligned}
& {\left[k_{s u}^{I}+M k_{s p}^{I}-\omega_{i}^{2} \frac{I_{s}^{I}}{\left(r_{s}^{I}\right)^{2}}\right] u_{s}^{I}+} \\
& M k_{s p}^{I}\left(u_{l}^{I}-\zeta_{1}^{I} \sin \alpha_{s m}-\eta_{1}^{I} \cos \alpha_{s m}\right) \\
& =0 \\
& \left(K_{r 2}^{I}\right)^{T} p_{r}^{I}+\left(K_{s 2}^{I}\right)^{T} p_{s}^{I}+ \\
& \left(K_{p m}^{I}-\omega_{i}^{2} M_{p}^{I}\right) p_{l}^{I}=0
\end{aligned}
$$

For each Eigen solution of this reduced eigenvalue problem, the full system mode is constructed from (8), (9) and (10). Generally, all the eigenvalues are distinct. Compared to the translational modes of planetary gears with equally spaced planets. These additional translational modes are discussed below.

\subsection{Planetary gear vibration modal analysis}

An interesting question is: when the planet spacing changes from equally spaced (with an even number of planets), how do the planet and purely ring modes, which exist only for equally spaced planets, evolve into rotational or translational modes? The rule is: If a mode for equally spaced planets has odd nodal diameter ring components, it evolves into a translational mode when the planets are diametrically opposed; if a mode has even nodal diameter ring components, it evolves into a rotational mode. To apply this rule, note that for equally spaced planets with even $\mathrm{N}$, the nodal diameter components of any mode are all even or all odd; for diametrically opposed planets, translational modes have all odd nodal diameter components while rotational modes have all even nodal diameter components. The vibration modal of planetary gear has the following characteristics.

Some clues guide the justification of the above rule. Every mode for equally spaced planets must evolve into either a rotational mode or a translational mode as the planets deviate to the diametrically opposed case.

Because of the continuity of the modes for changes in planet spacing, equally spaced modes retain this property when the planet spacing changes to diametrically opposed. One can imagine small deviations from equal spacing to clarify this continuity argument, but the conclusion is not restricted to that case; the properties established for small deviations must also hold for large deviations because the foregoing proof of the modal properties is not limited to small deviations [11].

$$
f_{i}=\left[\begin{array}{c}
0 \\
0 \\
0 \\
M \\
0 \\
p_{c}^{I I} \\
p_{r}^{I I} \\
p_{s}^{I I} \\
\left(\cos \psi_{1} p_{I}^{I}-\sin \psi_{1} \bar{p}_{1}^{I}\right) \\
M \\
\left(\cos \psi_{N} p_{I}^{I}-\sin \psi_{N} \bar{p}_{I}^{I}\right)
\end{array}\right]
$$




\section{International Journal of Science and Research (IJSR) \\ ISSN (Online): 2319-7064}

Index Copernicus Value (2013): 6.14 | Impact Factor (2014): 5.611

$$
\bar{f}_{i}=\left[\begin{array}{c}
0 \\
0 \\
0 \\
M \\
0 \\
\bar{p}_{c}^{I I} \\
\bar{p}_{r}^{I I} \\
\bar{p}_{s}^{I I} \\
M \\
\left(\sin \psi_{1} p_{1}^{I}+\cos \psi_{1} \bar{p}_{I}^{I}\right) \\
\left(\sin \psi_{N} p_{I}^{I}+\cos \psi_{N} \bar{p}_{1}^{I}\right)
\end{array}\right]
$$

When no vibration center component in planetary gear train, the vibration displacement sun gear is zero, when $p_{n}^{I I}=w_{n} p_{l}^{I I}$, where $w_{n}$ is the proportional coefficient, $w_{1}=1$, $n=1,2, \cdots N$.

This indicates planet modes, whether distinct or degenerate, having odd (even) nodal diameter components will evolve into translational (rotational) modes as the planets change from equally spaced.

The deflections of the planets (and all other rigid components) are zero for a purely ring mode. Thus, using the deflections of the planets as the condition to determine which type of mode it will evolve into does not work. A purely ring mode has one and only one nodal diameter component. For continuity of the modes, the mode that a purely ring mode evolves into should contain at least that specific nodal diameter component. Thus, if the purely ring mode has an odd nodal diameter component, the corresponding diametrically opposed mode will contain that (and other) odd nodal diameter components; this means the purely ring mode evolves into a translational mode. Similarly, if the purely ring mode has an even nodal diameter component, it evolves into a rotational mode.

Case 1: When $N / 2$ is odd, there are $(N-2) / 4$ even $M$ and $(N-6) / 4$ odd $M$ for $M$ in $[2, N / 2-1]$. Thus, $(N / 2-1)(2 M-3)$ degenerate planet modes contain even nodal diameter components, and they evolve into rotational modes; (N/2-3) $(2 M-3)+M-3$ planet modes evolve into translational modes, where $M-3$ modes come from the distinct planet modes.

According to Eq. (11) and Eq. (12), for even $N$ there is $M-3$ purely ring modes. When $N / 2$ is even, all the purely ring modes have an even nodal diameter component, thus they evolve into rotational modes.

\subsection{Coupled Vibration Modal Analysis}

For rotational modes of equally spaced planets, the deflections of all the planets are identical. Considering the coupled vibration mode characteristics, a rotational mode of equally spaced planets contains only nodal diameter components. Because $N$ is even, all the numbers of nodal diameter components are even.
When the star gear and planetary gear train are vibration, and the vibration of star gear train no rule, the inherent frequency of the system are single.

For translational modes of equally spaced planets, the planet deflections for a pair of translational coupling vibration modes satisfy.

$$
f_{i}=\left[p_{r}^{I}, p_{s}^{I}, p_{1}^{I}, \ldots, p_{M}^{I}, p_{c}^{I I}, p_{r}^{I I}, p_{s}^{I I}, p_{1}^{I I}, \ldots, p_{1}^{I I}\right]
$$

Where $p_{n}^{I I}=p_{1}^{I I}=\left[\zeta_{1}^{I I}, \eta_{1}^{I I}, u_{1}^{I I}\right]^{T}, p_{h}^{I I}=\left[0,0, u_{h}^{I I}\right], h=c, r, s$. Case 2: According to Eq. (13), coupling vibration modes of equally spaced planets remain translational modes. Because translational modes of equally spaced planets contain the nodal diameter components with even $N$, all the ring nodal diameter components are odd. Thus, one can identify it as a translational mode for equal space planets based on the even/odd condition as well.

In summary, for any mode of systems with equally spaced planets, whether the elastic ring nodal diameter components are even or odd determined.

\section{Conclusions}

This work analytically identifies the modal properties of planetary gears with equally spaced planets and sun gear. The discrete model represents the ring gear as an body free to deform radially while the remaining components are rigid. The elastic continuum ring model leads to an infinite-dimensional system. Relationships between the modal properties of planetary gears with equally spaced planets and sun gear are examined in detail. The following conclusions are obtained:

All the modes are classified into rotational or translational modes with distinct natural frequencies. Closed-form expressions are provided for the structure of each mode type. A rotational mode contains only even numbered nodal diameter components of the elastic ring, and a translational mode contains only odd numbered nodal diameter components. The planet and purely ring modes present when the planets and sun gear are equally spaced is exist.

For rotational modes, the translations for the ring rigid motion, sun, and carrier are zero. For translational modes, the rotations for the ring rigid motion, sun, and carrier are zero.

All the planet and purely ring modes of equally spaced planets evolve into either rotational or translational modes. The rule governing this modal evolution is: any mode for equally spaced planets and sun gear having odd (even) nodal diameter components evolves into a translational (rotational) mode.

\section{Acknowledgment}

This work is supported by pre-research project in Ship Research Institute of China (Grant Number: MAPT 41092013). Part of simulation works were performed on Dawning-TC5000 system in Supercomputing Centre, Shenzhen Institute of Advanced Technology, CAS, China. 


\section{International Journal of Science and Research (IJSR) \\ ISSN (Online): 2319-7064}

Index Copernicus Value (2013): 6.14 | Impact Factor (2014): 5.611

\section{References}

[1] J. Helsen, F. Vanhollenbeke, F. De Coninck, D. Vandepitte, W. Desmet. Insights in wind turbine drive train dynamics gathered by validating advanced models on a newly developed 13.2 MW dynamically controlled test-rig. Mechatronics, 21 (2011), pp. 737-752

[2] C.-J. Bahk, R.G. Parker. Analytical solution for the nonlinear dynamics of planetary gears. Journal of Computational and Nonlinear Dynamics, 2 (2011), p. 021007

[3] X. Wu, R.G. Parker. Modal properties of planetary gears with an elastic continuum ring gear. Journal of Applied Mechanics, 75 (2008), pp. 1-10

[4] R.G. Parker, X. Wu. Vibration modes of planetary gears with unequally spaced planets and an elastic ring gear. Journal of Sound and Vibration, 329 (2010), pp. 2265-2275

[5] Y. Guo, R.G. Parker. Purely rotational model and vibration modes of compound planetary gears. Mechanism and Machine Theory, 45 (2010), pp. 365-377

[6] C.G. Cooley, R.G. Parker. Unusual gyroscopic system eigenvalue behaviour in high-speed planetary gears. J. Sound Vib., 332 (2013), pp. 1820-1828

[7] D.R. Kiracofe, R.G. Parker. Structured vibration modes of general compound planetary gear systems. Journal of Vibration and Acoustics, 129 (2007), pp. 1-16

[8] C.G. Cooley, R.G. Parker. Vibration properties of high-speed planetary gears with gyroscopic effects. Journal of Vibration and Acoustics, 134 (2012), p. 061014

[9] V. Abousleiman, P. Velex, S. Becquerelle. Modeling of spur and helical gear planetary drives with flexible ring gears and planet carriers. Journal of Mechanical Design, 129 (2007), pp. 95-106

[10] V.K. Ambarisha, R.G. Parker. Nonlinear dynamics of planetary gears using analytical and finite element models. Journal of Sound and Vibration, 302 (2007), pp. 577-595

[11] A. Singh, A. Kahraman, H. Ligata. Internal gear strains and load sharing in planetary transmissions: model and experiments. Journal of Mechanical Design, 130 (2008), p. 072602

[12] Y. Guo, R.G. Parker. Stiffness matrix calculation of rolling element bearings using a finite element/contact mechanics model. Mechanism and Machine Theory, 51 (2012), pp. 32-45

[13] C.-J. Bahk, R.G. Parker. Analytical solution for the nonlinear dynamics of planetary gears. Journal of Computational and Nonlinear Dynamics, 2 (2011), p. 021007

[14] M. Kang, A. Kahraman. Measurements of vibratory motions of gears supported by compliant shafts. Mechanical Systems and Signal Processing, 29 (2012), pp. 391-403

[15] R.M.H. Khorasany, S.G. Hutton. The effect of axisymmetric nonflatness on the oscillation frequencies of a rotating disk. Journal of Vibration and Acoustics, 132 (2010), p. 051012
[16] T.E. Ericson, R.G. Parker. Planetary gear modal vibration experiments and correlation against lumped-parameter and finite element models. J. Sound Vib., 332 (2013), pp. 2350-2375

[17] C.G. Cooley, R.G. Parker. Vibration properties of high-speed planetary gears with gyroscopic effects. ASME J. Vib. Acoust., 134 (2012), p. 061014

[18] T. Eritenel, R.G. Parker. Modal properties of three-dimensional helical planetary gears. Journal of Sound and Vibration, 325 (2009), pp. 397-420

[19] C.-J. Bahk, R.G. Parker. Analytical solution for the nonlinear dynamics of planetary gears. ASME J. Comput. Nonlinear Dyn., 6 (2011), p. 021007

[20] X. Gu, P. Velex. A dynamic model to study the influence of planet position errors in planetary gears. J. Sound Vib., 331 (2012), pp. 4554-4574

[21] M. Inalpolat, A. Kahraman. A dynamic model to predict modulation sidebands of a planetary gear set having manufacturing errors. J. Sound Vib., 329 (2010), pp. 371-393

[22] R. Dhanasekaran, P.S. Kumar, K. Santhi. Crack failure of planetary gearbox sun gear. Int J Recent Trends Eng Technol, 3 (6) (2010), pp. 12-14

\section{Author Profile}

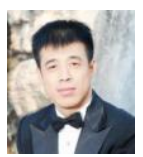

Xigui Wang was born in China on May 24, 1972, I grew up in Harbin Institute of Technology where I obtained my bachelor's and master's degree. My 20 year-plus academic career evolved at the Chinese Ship Power Transmission Systems Research Institute and the Mechatronics school (Harbin Institute of Technology) as Professor until now. 